\title{
Geometric Finiteness and Uniqueness for Kleinian Groups with Circle Packing Limit Sets
}

\author{
Linda Keen* \\ Mathematics Department \\ CUNY Lehman College \\ Bronx, NY 10468, U.S.A. \\ Bernard Maskit ${ }^{\dagger}$ \\ Mathematics Department \\ SUNY Stony Brook \\ Stony Brook, NY 11794-3561, U.S.A. \\ Caroline Series \\ Mathematics Institute \\ Warwick University \\ Coventry CV4 7AL, U.K.
}

August 30, 2018

Let $G \subset P S L(2, \mathbf{C})$ be a geometrically finite Kleinian group, with region of discontinuity $\Omega(G)$. By Ahlfors' finiteness theorem, the quotient, $\Omega(G) / G$, is a finite union of Riemann surfaces of finite type. Thus on it, there are only finitely many mutually disjoint free homotopy classes of simple closed curves. It is shown in [9] and [13] that if $\gamma_{1}, \ldots, \gamma_{k}$ are a set of mutually disjoint and simple closed curves in $\Omega(G) / G$, represented by primitive non-elliptic and non-conjugate elements $g_{1}, \ldots, g_{k}$ of $G$, then there is a group $G^{\prime}$, and an isomorphism $\phi: G \rightarrow G^{\prime}$ taking parabolic elements of $G$ to parabolic elements of $G^{\prime}$, for which the images $\phi\left(g_{1}\right), \ldots, \phi\left(g_{k}\right)$ in $G^{\prime}$ are parabolic. Heuristically, this means that the curves $\gamma_{i}$ have been "shrunk" or "pinched" to punctures.

In this paper, we assume that $G$ is a finitely generated torsion free non-elementary Kleinian group with $\Omega(G) \neq \emptyset$. We show that the maximal number of elements of $G$ that can be pinched is precisely the maximal number of rank 1 parabolic subgroups that any group isomorphic to $G$ may contain. A group with this largest number of rank 1 maximal parabolic subgroups is called maximally parabolic. We show such groups exist. We state our main theorems concisely here. Full statements appear in sections 4 and 5 .

Theorem I. The limit set of a maximally parabolic group is a circle packing; that is, every component of its regular set is a round disc.

and

Theorem II. A maximally parabolic group is geometrically finite.

We define a class of groups called pinched function groups. Roughly speaking, these are either function groups, or groups isomorphic to function groups, for which a set of loxodromic elements has been pinched to parabolic ones. The precise definition is given in section 5 .

Theorem III. A maximally parabolic pinched function group is determined up to conjugacy in $\operatorname{PSL}(2, \mathbf{C})$ by its abstract isomorphism class and its parabolic elements.

\footnotetext{
*Supported in part by NSF GRANT DMS-8902881

${ }^{\dagger}$ Supported in part by NSF GRANT DMS-9003361
} 
It is a well known consequence of Jørgensen's inequality that if $G$ is a non-elementary geometrically finite function group, and $\mathcal{T}(G)$ is its quasi-conformal deformation space, then any group in $\partial \mathcal{T}(G)$ is discrete. Let $g_{1}, \ldots, g_{k}$ be elements of $G$ which represent a maximal set of mutually disjoint and non-homotopic simple closed curves on $\Omega(G) / G$. An application of our results is that there exists a unique point in $\partial \mathcal{T}(G)$ for which all the elements $\left(g_{i}\right), i=1, \ldots, k$, are parabolic.

An outstanding conjecture in the theory of Kleinian groups (see for example, [17]) is that groups on the boundary of certain円 deformation spaces of Kleinian groups are uniquely determined by their ending laminations. Our result proves this conjecture in a special case.

A well studied deformation space of Kleinian groups is the deformation space of free groups on two generators. This is the Schottky space of genus 2; for $G$ in this space $\Omega(G) / G$ is a surface $S$ of genus 2. The space has three complex dimensions and has a complicated boundary. A one dimensional slice of this boundary is formed by shrinking two curves on $S$, one a dividing curve and the other a non-dividing curve. The resulting surfaces consist of a punctured torus and a triply punctured sphere. Since the triply punctured sphere has a unique conformal structure, this boundary slice is an embedding of the Teichmüller space of a punctured torus. It is known as the Maskit embedding [8]. Wright [19] studied the boundary of this embedding computationally and conjectured that the cusp groups for which an element corresponding to a particular simple curve on the torus is parabolic were unique. Our theorem proves this conjecture.

Another one dimensional boundary slice of the Schottky space of genus 2 is formed by shrinking two non-dividing curves on $S$. The resulting surfaces are spheres punctured at four points and the resulting groups are generated by two parabolics. Groups generated by two parabolics were investigated computationally by Riley [14] and by Maskit and Swarup [11]; this boundary slice is known as the Riley slice. Our theorem proves that the cusp groups on the boundary of the Riley slice for which an element corresponding to a particular simple closed curve on the four punctured sphere is parabolic are unique.

On each of these boundaries, three elements of $G$ were specified to be parabolic and this determined the group uniquely. It also follows from our uniqueness theorem that if the same three elements are parabolic at a boundary point of a Maskit slice and at a boundary point of a Riley slice, then they correspond to the same group on the boundary of Schottky space. We refer the reader to [3, 4] and the references therein for further discussion of these spaces.

The method we use for the proofs of theorems I and II is an extension of that developed by Maskit and Swarup in [11] where they prove theorem II in the special case that $G$ is generated by two parabolic elements. The method depends crucially on the fact that any hyperbolic manifold has a compact core that supports all the homotopy. For theorem III we study the deployment of the circles in the limit set and then apply Marden's isomorphism theorem [5].

The outline of this paper is as follows. Section 1 contains basic definitions and notation and a summary of the results we need about cores of hyperbolic 3-manifolds. We discuss cusp cylinders and pairing tubes associated to cyclic parabolic subgroups. In section 2, we define an invariant of the isomorphism class of $G$, called the boundary characteristic of a Kleinian group, in terms of the Euler characteristic of the boundary of the core. The boundary characteristic determines how many loops may be pinched. In section 3 we discuss maximally parabolic groups and prove that the isomorphism class of any geometrically finite group contains a group that is maximally parabolic. In section 4 we prove theorems I and II. The exact definition of pinched function groups is given in section 5 and theorem III is proved there.

We would like to thank Jean-Pierre Otal and Colin Rourke for helpful discussions about this paper. The second author thanks the Institut des Hautes Etudes Sciéntifique and the last author

\footnotetext{
${ }^{1} \mathrm{~A}$ suitable condition is that the components of the boundary of a compact core of $\mathbf{H}^{3} / G$ are incompressible.
} 
thanks the M.S.I. at Stony Brook for their hospitality.

\section{Preliminaries}

\subsection{Basics.}

A Kleinian group $G$ is a discrete subgroup of $P S L(2, \mathbf{C})$. It acts by conformal automorphisms on the Riemann sphere $\hat{\mathbf{C}}$ and by isometries on hyperbolic three space $\mathbf{H}^{3}$. We denote by $\Omega=\Omega(G) \subset \hat{\mathbf{C}}$ the region of discontinuity of $G$, i.e. the set on which the elements of $G$ form a normal family, and by $\Lambda=\Lambda(G)$ its complement. Throughout this paper, we make the hypotheses, which we shall not restate, that all our Kleinian groups are non-elementary, finitely generated, torsion free and that $\Omega(G) \neq \emptyset$.

A component of the group $G$ is a connected component of $\Omega$ and a subgroup of $G$ stabilising a component is called a component subgroup. We denote the quotient $\mathbf{H}^{3} \cup \Omega(G) / G$ by $M=M(G)$. The group $G$ is called geometrically finite if it has a finite sided convex fundamental polyhedron in $\mathbf{H}^{3}$.

In what follows we shall frequently need to make use of a more refined notion of invariance called precise invariance, (see [10]). Namely, let $H$ be a subgroup of $G$; a subset $E \subset \Omega \cup \mathbf{H}^{3}$ is said to be precisely invariant under $H$ in $G$ if $E$ is invariant under $H$ and if $g(E) \cap E=\emptyset$ whenever $g \notin H$. More generally, a family of sets $E_{1}, \ldots, E_{n}$ is precisely invariant under a family of subgroups $H_{1}, \ldots, H_{n}$ if each $E_{i}$ is invariant under $H_{i}$ and if, for any $g \in G, g\left(E_{i}\right) \cap E_{j} \neq \emptyset$ only if $i=j$ and $g \in H_{i}=H_{j}$.

\subsection{Parabolic cusps and cores}

Suppose that $H$ is a maximal parabolic subgroup of rank one in a Kleinian group $G$; there is a single point $p$ fixed by all its elements. Let $C$ be an open horoball (i.e. an open Euclidean 3-ball) tangent to $\partial \mathbf{H}^{3}$ at $p$. Clearly $C$ is $H$-invariant and in fact, (see e.g. 10 p.120), one may choose $C$ to be precisely invariant under $H$. By putting $p$ at infinity, and using the precise invariance, one sees easily that $\partial C / G$ is topologically an open annulus if $H$ has rank 1 , while it is a torus if $H$ has rank 2.

Suppose that $G$ contains $d$ non-conjugate maximal parabolic subgroups $H_{1}, \ldots, H_{d}$. For each $i=1, \ldots, d$, let $C_{i}$ be an open horoball tangent to $\partial \mathbf{H}^{3}$ at the fixed point of $H_{i}$. Such a $C_{i}$ is automatically $H_{i}$ invariant and in fact, (see [10] pp. 119-120), one can choose the set $\left(C_{1}, \ldots, C_{d}\right.$ ) to be mutually disjoint and precisely invariant under $\left(H_{1}, \ldots, H_{d}\right)$ in $G$. It follows from the precise invariance that the $C_{i}$ all have disjoint projections, and that the boundaries of $C_{i} / G$ are either tori or compact cylinders. We call the corresponding $C_{i} / G$ cusp tori and cusp cylinders respectively. Let $\tilde{N}(G)=\mathbf{H}^{3}-\cup_{i=1}^{d} C_{i}$. Then $N(G)=\tilde{N}(G) / G=M(G)-\{\}$ cusp tori $\} \cup\{$ cusp cylinders $\}$.

If $G$ is a Kleinian group containing parabolics, then $M(G)$ certainly is not compact. A core $K$ of a 3-manifold $M$ is a compact connected submanifold of $M$ with the property that the injection $K \rightarrow M$ induces an isomorphism of fundamental groups. It was shown by Scott [15] that every 3-manifold with finitely generated fundamental group has a core; this was refined by McCullogh [12], who showed that the core can be chosen so that it intersects the boundary of $M$ in any predetermined compact submanifold of $\partial M$.

If we apply these results to $N(G)$ we see that we can choose a core $K(G)$ for $N(G)$ so that its boundary contains the cusp tori and cusp cylinders. Since there exists a retraction from $M(G)$ to $N(G)$, any core for $N$ is automatically a core for $M$. Notice that because we are in hyperbolic space the core can be chosen to be irreducible. 


\subsection{Pairing tubes}

When we have a cusp cylinder, in general we know nothing about the action of $G$ in the neighbourhood of a parabolic fixed point outside the horoball neighbourhood $C$. However, there is one important case in which we have much more precise information. Namely, suppose that there are two disjoint circular discs, $D_{1}, D_{2} \subset \Omega$, mutually tangent at $p$, whose union is precisely invariant under the subgroup $H$ fixing $p$. Then the parabolic point $p$ is said to be doubly cusped. The two hyperbolic half spaces, $\Sigma_{1}$ and $\Sigma_{2}$ above $D_{1}$ and $D_{2}$, in $\mathbf{H}^{3}$ are also precisely invariant, and the quotient $\Sigma_{1} \cup C \cup \Sigma_{2} / G$ is a cylinder with two ends at infinity, as described in detail in [5] Sec 2.6 or [1] Lemma 4. The quotient cylinder in this set-up is called a pairing tube.

Definition 1.3.1. The natural truncation $T(G)$ of $M(G)$ is the manifold obtained by removing the pairing tubes and cusp tori from $M(G)$.

The natural truncation is clearly a retraction of $M(G)$. The boundary of $T$ is the union of the boundaries of the cusp tori and components formed from the boundary components of $\Omega / G$ as follows. We remove a punctured disc neighbourhood from each double cusp and then, for each pair of discs, we glue in an annulus joining the boundary of one disc at this cusp to the other. In this way we obtain the boundary of a cusp cylinder. Note that the boundary of $T$ is compact if and only if all the rank 1 parabolic points are doubly cusped.

Remark 1.3.2. It follows from [5] that if $G$ is geometrically finite then all the rank 1 parabolic fixed points are doubly cusped. Hence we can restate Marden's criterion for geometric finiteness in terms of $T(G)$ as: $G$ is geometrically finite if and only if $T(G)$ is compact.

\section{Boundary characteristic and geometric finiteness}

Proposition 2.1. The maximal number of distinct disjoint simple closed curves on the boundary of a core of $M(G)$ depends only on the isomorphism class of $G$. In particular, it is independent of the choice of core.

Proof. Let $G$ and $G^{\prime}$ be isomorphic finitely generated Kleinian groups. Let $K(G)$ and $K\left(G^{\prime}\right)$ be cores of $G$ and $G^{\prime}$ chosen as in section 1.2.

Note that $K(G)$ and $K\left(G^{\prime}\right)$ are $K(\pi, 1)^{\prime} s$ with isomorphic fundamental groups, and hence are homotopy equivalent. In particular $K(G)$ and $K\left(G^{\prime}\right)$ have the same Euler characteristic. Since the Euler characteristic of a closed orientable 3-manifold is zero, the Euler characteristic of the boundary of a compact orientable 3-manifold is twice the Euler characteristic of the manifold itself. Hence the boundaries of $K(G)$ and $K\left(G^{\prime}\right)$ have the same Euler characteristic, say $2 \chi$.

As remarked above, we may assume neither core has a boundary component that is a sphere. Since the groups $G$ and $G^{\prime}$ are isomorphic, and the tori on the boundary of the core correspond exactly to the conjugacy classes of maximal rank 2 parabolic subgroups (equivalently rank 2 abelian), $K(G)$ and $K\left(G^{\prime}\right)$ have the same number $\tau$ of tori on their boundaries.

The maximal number of non-homotopic disjoint simple closed curves on a union of surfaces of negative Euler characteristic is exactly $-3 \chi$. Hence, for both $K(G)$ and $K\left(G^{\prime}\right)$, the number of disjoint homotopically distinct simple loops on their boundaries is $\tau-3 \chi$.

Definition 2.2. The boundary characteristic $b=b(G)$ of the Kleinian group $G$ is $n-\tau$ where $n$ is the maximal number of disjoint homotopically distinct simple closed curves on the boundary of any core for $G$, and $\tau$ is the number of conjugacy classes of rank 2 maximal parabolic subgroups of $G$. 
We can give a criterion for geometric finiteness of $G$ in terms of the boundary characteristic and the truncation $T(G)$ of $M(G)$.

Proposition 2.3. Let $G$ be a Kleinian group. If $G$ is geometrically finite then the non-toral components of $\partial T(G)$ support $b(G)$ homotopically distinct disjoint simple closed curves. The converse is also true if, in addition, all rank one parabolic fixed points are doubly cusped.

Proof. If $G$ is geometrically finite, then by remark 1.3 .2 the natural truncation $T$ is compact. Since $T$ is a retraction of $M$, we may take it to be a core $K$ of $M$; hence it supports at most $b$ distinct simple closed curves on the non-toral components.

Suppose conversely that the non-toral part of $\partial T$ supports $b$ distinct simple closed curves, and that every rank one parabolic fixed point is doubly cusped. Let $\Omega_{1}$ be the set obtained from $\Omega$ by removing disc neighbourhoods of the doubly cusped parabolic points. The boundary $\partial T$ is the union of the boundaries of the pairing tubes, the cusp tori and the components of $\Omega_{1} / G$. Using the assumption about the rank one parabolic points, we see that $\partial T$ is compact. Thus we may choose a compact core $K$ for $M$ whose boundary contains $\partial T$ and such that $K \subset T$. By proposition 2.1, the boundary $\partial K$ of $K$ also supports $b$ disjoint simple closed curves on its non-toral part. Now $\partial K$ and $\partial T$ contain the same number of cusp tori so $\partial T=\partial K$ and hence $K=T$. Hence $T$ is compact and so Marden's criterion for geometric finiteness is satisfied (see [5] and also [10] pp. 128-130).

Remark. Observe that if $G$ is geometrically finite and purely loxodromic, then $T=M, \partial T=\partial M$ and $b$ is the maximal number of disjoint homotopically distinct simple closed curves that one can draw on $\Omega / G$.

\section{Boundary characteristic and parabolics}

Proposition 3.1. The number of conjugacy classes of rank 1 maximal parabolic subgroups in a Kleinian group $G$ is at most $b(G)$.

Proof. Form the core $K$ as described in section 1.2. For each cusp cylinder in $\partial K$ we have a simple loop, and these loops are disjoint and homotopically distinct. Since there are at most $b$ such cylinders we are done.

Definition 3.2 A Kleinian group $G$ that contains $b(G)$ distinct conjugacy classes of rank 1 maximal parabolic subgroups is called maximally parabolic.

As an immediate corollary to proposition 3.1 we have:

Corollary 3.3. If $G$ is maximally parabolic, then, on any core $K(G)$ there are $b(G)$ disjoint homotopically distinct simple closed curves on non-toral components of $\partial K(G)$, each of which is represented by a parabolic element of $G$. These $b(G)$ simple loops divide the non-toral part of $\partial K(G)$ into $2 b(G) / 3$ pairs of pants.

The existence of maximally parabolic groups follows from the following theorem.

Theorem 3.4. Let $G$ be a geometrically finite Kleinian group. Then there exists a maximally parabolic group $G^{\prime}$ and an isomorphism $\phi: G \rightarrow G^{\prime}$ mapping parabolic elements of $G$ to parabolic elements of $G^{\prime}$.

The essential ingredient in the proof is the following theorem.

Theorem 3.5. Let $G$ be a geometrically finite Kleinian group and let $g \in G$ be a loxodromic element representing a simple closed loop on some component of $\Omega / G$. Then there exists a geometrically finite Kleinian group $G^{\prime}$, and an isomorphism $\phi: G \rightarrow G^{\prime}$ that maps parabolic elements of $G$ to parabolic elements of $G^{\prime}$, and such that $\phi(g)$ is parabolic. 
This theorem was proved by Maskit 9] for function groups and the proof is easily extended to the class of pinched function groups defined in section 5. Based on an extension of theorem 7.1 in [18], Ohshika, [13], generalised this result to all geometrically finite Kleinian groups.

We shall also need the following lemma.

Lemma 3.6. Let $G$ be a Kleinian group and suppose that some component $S$ of $\Omega / G$ is not a triply punctured sphere. Then there is a loxodromic element in $G$ that represents a simple closed curve on $S$.

Remark. This lemma is closely related to proposition 2 in [13].

Proof. Let $\Omega_{0}$ be a component of $\Omega$ that is a lift of the surface $S$. The component subgroup $H$ stabilising $\Omega_{0}$ is non-elementary since its limit set $\partial \Omega_{0}$ is a subset of the perfect set $\Lambda(G)$. Thus, $H$ is a function group with invariant component $\Omega_{0}$ containing infinitely many loxodromic elements, all of which represent loops on $S$. Assume that none of these is simple.

Using combination theorem techniques, it was shown in [7] (see also [10], chapter X) that $H$ is a free product of a finite number of subgroups $H_{1}, \ldots, H_{n}$, where each factor is one of three possible types. The factor may be a group with a simply connected invariant component; in this case, we have a universal cover for a subsurface of $S$. It may be an elementary group with one limit point; then it is either a rank 1 or rank 2 parabolic subgroup. Finally, it may be a purely loxodromic free group (i.e. a Schottky group).

One of the conclusions of the free product combination theorem is that one may obtain a fundamental domain for $H$ from fundamental domains for the factors $H_{i}$ by cutting and pasting fundamental domains for the $H_{i}$ in such a way that if $h \in H_{i}$ represents a simple loop on $\Omega\left(H_{i}\right) / H_{i}$, then $h$ represents a simple loop on $S$. We may therefore assume that no loxodromic element of any $H_{i}$ represents a simple loop.

The quotient surface of a purely loxodromic free group is a compact surface and each generator represents a simple loop. Hence, by our assumption, none of the factors $H_{i}$ are of this type.

It was shown in [7] that if $H_{i}$ has a simply connected invariant domain, then the set of accidental parabolic elements represents a finite set of simple disjoint homotopically distinct loops on its quotient surface. Either there are infinitely many simple loops and so most are represented by loxodromic elements or $H_{i}$ is a Fuchsian group with quotient a triply punctured sphere.

Since $H$ is non-elementary, and itself not a triply punctured sphere group, we conclude that the free product description of $H$ has at least two factors and that the factors are either distinct rank 1 or rank 2 parabolic subgroups or triply punctured sphere groups. In the cut and paste construction of fundamental domains, for each pair of factor subgroups, there is a homotopically nontrivial loop, say $\tilde{\gamma}$, so that fundamental domains for this pair of factors are pasted across $\tilde{\gamma}$. This loop projects to a simple homotopically non-trivial loop $\gamma$ on $S$. Since $S$ is hyperbolic of finite area, and not a triply punctured sphere, there is a simple homotopically non-trivial loop on $S$ crossing $\gamma$ that is represented by a word of length at least two (using the free product word length) in $H$. Another conclusion of the free product combination theorem is that every parabolic element in $G$ is conjugate to a parabolic element of some factor. It follows, therefore, that the element we have found is loxodromic.

Proof of Theorem 3.4 Let $G$ be a geometrically finite Kleinian group that is not maximally parabolic. Suppose that it contains $p(G)<b(G)$ distinct conjugacy classes of rank 1 parabolic subgroups. We shall show that there is a group $G^{\prime}$ and an isomorphism $\phi: G \rightarrow G^{\prime}$, taking parabolics to parabolics, such that $G^{\prime}$ has $p\left(G^{\prime}\right)>p(G)$ distinct conjugacy classes of rank 1 parabolic subgroups. Since we can repeat this argument until the number of such classes is equal to $b(G)$, this will prove the proposition. 
Since $G$ is geometrically finite, by remark 1.3.2 the truncation $T(G)$ is compact and so can be taken as a core $K(G)$ for $M(G)$. By proposition 2.3 we can find exactly $b(G)$ disjoint simple closed loops on the non-toral part of $\partial T(G) ; p(G)$ of these can be taken to be loops around the boundaries of the cusp cylinders. If all the components of $\Omega / G$ were triply punctured spheres it would be impossible to find any more disjoint loops on $\partial T(G)$ so that $p(G)=b(G)$; this is not the case.

Let $S$ be a component of $\Omega / G$ that is not a triply punctured sphere. By lemma 3.6 we can find a loxodromic element $g \in G$ representing a simple closed curve on $S$ and by theorem 3.5 there is a geometrically finite group $G^{\prime}$ and an isomorphism $\phi: G \rightarrow G^{\prime}$, taking parabolics to parabolics and such that $\phi(g)$ is also parabolic. Therefore $G^{\prime}$ has at least one more conjugacy class of rank 1 parabolic subgroups than $G$ as required.

\section{Proofs of theorems I and II}

In this section we prove the following two related results. We recall that $G$ is non-elementary, finitely generated, torsion free and has a non-empty regular set.

Theorem I. Let $G$ be a maximally parabolic Kleinian group $G$ of boundary characteristic $b(G)$. Then $\Omega(G)$ is a union of round discs and $\Omega(G) / G$ is a union of triply punctured spheres. For each maximal parabolic subgroup $H \subset G$ of rank 1, exactly two of these round discs are tangent at the fixed point of $H$; in particular, all parabolic fixed points are doubly cusped.

Theorem II. A maximally parabolic Kleinian group $G$ is geometrically finite.

An immediate corollary is:

Corollary 4.1. Let $G$ be a geometrically finite Kleinian group of boundary characteristic $b(G)$. Let $\phi$ be an isomorphism onto a subgroup $\phi(G)$ of $P S L(2, \mathbf{C})$, where $\phi(h)$ is parabolic for every parabolic $h \in G$, and $\phi(G)$ has $b(G)$ distinct conjugacy classes of rank 1 maximal parabolic subgroups. Then $\phi(G)$ is either not discrete, or it is discrete with non-empty regular set and is geometrically finite.

Proof of theorem I. Let $b$ be the boundary characteristic of $G$, let $K$ be a core for $M$ as in section 1.2 , and let $C$ denote the union of components of $\partial K$ that are not cusp tori. By corollary 3.3 there are b disjoint homotopically distinct simple loops on $C$ representing distinct parabolic elements of $G$. These divide $C$ into $2 \mathrm{~b} / 3$ pairs of pants.

Let $P$ be one of these pairs of pants and let $\gamma_{1}, \gamma_{2}, \gamma_{3}$ be loops going around the boundary curves of $P$, chosen so that $\gamma_{1} \gamma_{2} \gamma_{3}=i d$ in $\pi_{1}(P)$. Each $\gamma_{i}$ lies on the boundary of a cusp cylinder corresponding to a conjugacy class of maximal parabolic subgroups in $G$; hence each $\gamma_{i}$ bounds a punctured disc contained in the cusp cylinder. Adjoining these punctured discs to $P$ we obtain a triply punctured sphere $Q$.

Lift $Q$ to $\mathbf{H}^{3}$ and let $\tilde{Q}$ be a connected component of this lift. Let $R \subset \tilde{Q}$ be a simply connected fundamental domain for the action of $G$ on $\tilde{Q}$, such that the Euclidean closure of $R$ in $\hat{\mathbf{C}}$ consists of four parabolic fixed points $\xi_{1}, \xi_{2}, \xi_{3}, \xi_{4}$ on $\hat{\mathbf{C}}$. We label these so that there is a connected component of the lift $\tilde{\gamma}_{i}$ of $\gamma_{i}$ with both endpoints at $\xi_{i}, i=1,2,3$. We can choose generators, $h_{i}$ of the parabolic subgroups stabilising $\xi_{i}$, such that $h_{1} h_{2} h_{3}=1$ in $G$. An easy computation with matrices now shows that $F=<h_{1}, h_{2}, h_{3}>$ is Fuchsian, and that its limit set is a circle $\Lambda(F)$.

By comparing the action of $F$ on $R$ with its action on a standard fundamental domain in the Poincaré disc, we see that $\cup_{f \in F} f(R)$ is both open and closed in $\tilde{Q}$. Therefore, $\tilde{Q}=\cup_{f \in F} f(R)$. It follows that the boundary of $\tilde{Q}$ in $\mathbf{H}^{3} \cup \hat{\mathbf{C}}$ is exactly $\Lambda(F)$.

Since $Q=R / G=\tilde{Q} / F$ is a triply punctured sphere, we see that $F=\pi_{1}(\tilde{Q} / F)$. Consequently, $\tilde{Q}$ is a universal cover for $Q$. It follows that $\tilde{Q}$ is a topological disc in $\mathbf{H}^{3}$ whose boundary in $\mathbf{H}^{3} \cup \hat{\mathbf{C}}$ 
is exactly $\Lambda(F)$. Thus $\tilde{Q}$ separates $\mathbf{H}^{3}$.

Now let $\tilde{K}$ be the lift of the core $K$ to $\mathbf{H}^{3}$. Since $K$ is connected so is $\tilde{K}$; also, since $Q \subset \partial K$, we know that $\tilde{Q} \subset \partial \tilde{K}$. It follows that $\tilde{K}$ is entirely contained on one side of $\tilde{Q}$. Denote by $\Delta(F)$ the open disc in $\hat{\mathbf{C}}$ bounded by $\Lambda(F)$ and on the opposite side of $\tilde{Q}$ from $\tilde{K}$. Because $\tilde{K}$ is $G$-invariant, there can be no limit points of $G$ in $\Delta(F)$.

We next show that $\Delta(F)$ is precisely invariant under $F$. Clearly, any image of $\Delta(F)$ that intersects $\Delta(F)$ coincides with it, so we have only to show that the component subgroup $F_{\Delta}$ of $\Delta$ is just $F$. Temporarily think of $\Delta(F)$ as a hyperbolic disc in which $F_{\Delta}$ and $F$ act as Fuchsian groups. If $F_{\Delta} \supset F, F_{\Delta} \neq F$, then $\Delta(F) / F \rightarrow \Delta(F) / F_{\Delta}$ would be a covering of hyperbolic surfaces of degree greater than 1 , and so $\Delta(F) / F_{\Delta}$ would be a surface of hyperbolic area less than $2 \pi$. Since $G$, and hence $F_{\Delta}$, contain no elliptics, this is impossible.

Suppose now that $P$ and $P^{\prime}$ are distinct pairs of pants adjacent along the same curve on $\partial K$. Then the circles $\Lambda(F)$ and $\Lambda\left(F^{\prime}\right)$ constructed above are distinct and are both invariant under a common parabolic element $h$ of $G$, so they are mutually tangent. Since the disks $\Delta$ and $\Delta^{\prime}$ do not intersect, the parabolic subgroup generated by $h$ is doubly cusped in the sense of section 1.3. It is clear that the fixed point of every cyclic parabolic subgroup is such a point of mutual tangency and so doubly cusped.

Finally, we want to show that the union of the discs $\Delta_{i}$ for each of the component subgroups $F_{i}$ is all of $\Omega$; i.e. $\Omega=\cup_{i} \Delta_{i}$. Adjoin to $K$ the portions of the cusp cylinder bounded between the punctured discs that we adjoined to the pants in $\partial K$ to make the triply punctured spheres $Q_{i}$, and call the resulting region $A$. Then the lift $\tilde{A}$ of $A$ to $\mathbf{H}^{3}$ is connected and its boundary in $\mathbf{H}^{3}$ is exactly the union of all the lifts $\tilde{Q}_{i}$ of the triply punctured spheres $Q_{i}$.

Choose $\eta \in \Omega$, and pick an oriented geodesic $\beta$ running from some point in $\tilde{A}$ to $\eta$. Let $\xi$ be the last point of $\beta$ in $\tilde{A}$. Then $\xi$ lies in one of the surfaces $Q_{i}$. Since by the choice of $\xi$ the segment of $\beta$ from $\xi$ to $\eta$ does not recross $\partial \tilde{A}$, it is easy to see that $\eta$ lies in a disc $\Delta_{i}$ as required.

Proof of Theorem II. By Theorem I we know that all the rank 1 parabolic fixed points are doubly cusped. It follows that there are the maximal number, $b$, of simple closed loops on $\partial T$, and hence, by the finiteness criterion of proposition 2.3 , that $G$ is geometrically finite.

\section{$5 \quad$ Pinched function groups and uniqueness}

We shall prove our uniqueness result for a restricted class of groups that we call pinched function groups.

Let $G$ be a Kleinian group and let $\left\{\Omega_{i}\right\}$ be the components of $\Omega$. We say $\Omega_{i}$ is adjacent to $\Omega_{j}$ if there is a doubly cusped parabolic element in $G$ with invariant $\operatorname{discs} D \subset \Omega_{i}$ and $D^{\prime} \subset \Omega_{j}$. We define an equivalence relation on $\left\{\Omega_{i}\right\}$ by $\Omega_{i} \equiv \Omega_{j}$ if and only if there is a finite sequence of components $\Omega_{i}=\Omega_{n_{1}}, \Omega_{n_{2}}, \ldots \Omega_{n_{d}}=\Omega_{j}$ with $\Omega_{n_{k}}$ adjacent to $\Omega_{n_{k+1}}, k=1, \ldots, d-1$.

We call the union of all the $\Omega_{i}$ in an equivalence class an augmented component of $G$.

Definition 5.1. A Kleinian group $G$ is a pinched function group if it has a $G$-invariant augmented component.

Remark. The augmented components of a Kleinian group $G$ are in one to one correspondence with the non-toral components of $\partial T$, the boundary of the natural truncation $T$ of $M$.

It follows that if $G$ is a geometrically finite pinched function group and if $G^{\prime}$ is obtained from $G$ by pinching, then $G^{\prime}$ is also a pinched function group.

We can now state our uniqueness theorem. 
Theorem III. Let $G$ and $G^{\prime}$ be maximally parabolic Kleinian groups where $G$ is a pinched function group. Suppose that there is an isomorphism $\phi: G \rightarrow G^{\prime}$ where $\phi(g)$ is parabolic if and only if $g$ is parabolic. Then there is a (possibly orientation reversing) Möbius transformation $\alpha$, so that $\phi(g)=\alpha \circ g \circ \alpha^{-1}$, for all $g \in G$.

Before we can proceed with the proof we need some preliminaries. We suppose that $G, G^{\prime}$ and $\phi$ are as in the statement of Theorem III.

Let $\mathcal{F}(G)$ denote the set of Fuchsian subgroups of $G$ with three parabolic generators whose product is the identity. Any group $H$ in $\mathcal{F}(\mathcal{G})$ has a circular limit set contained in $\Lambda(G)$. However, as can be seen from Example VIII.G.1 in 10 there are, at least for some groups $G$, elements of $\mathcal{F}(G)$ whose limit circles have limit points of $G$ both inside and outside. We denote the subset of $\mathcal{F}(G)$ consisting of those subgroups which are also component subgroups of $G$ by $\mathcal{F}_{0}(G)$. We call such groups peripheral.

Note that if $H \in \mathcal{F}_{0}(G)$, then the the limit set of $H$ is a circle bounding an open disc $\Delta(H)$ in $\Omega(G)$.

It is clear from the definition that an isomorphism $\phi$ such as the one in theorem III maps the groups in $\mathcal{F}(G)$ onto the groups in $\mathcal{F}\left(G^{\prime}\right)$.

Proposition 5.2. If $G$ is a pinched function group then the isomorphism $\phi$ maps the groups in $\mathcal{F}_{0}(G)$ onto those in $\mathcal{F}_{0}\left(G^{\prime}\right)$.

The main ingredient in the proof of this proposition is the following theorem of Susskind [16]:

Theorem 5.3 (Susskind). Let $H_{1}$ and $H_{2}$ be geometrically finite subgroups of a (torsion free) Kleinian group $G$. Then

$$
\Lambda\left(H_{1}\right) \cap \Lambda\left(H_{2}\right)=\Lambda\left(H_{1} \cap H_{2}\right) .
$$

An immediate corollary of theorem 5.3 is:

Corollary 5.4. Let $G$ be a maximally parabolic Kleinian group and let $F_{1}, F_{2} \in \mathcal{F}_{0}(G)$. Then the circles $\Lambda\left(\phi\left(F_{1}\right)\right)$ and $\Lambda\left(\phi\left(F_{2}\right)\right)$ are either tangent or disjoint. In the first case, $F_{1} \cap F_{2}$ consists of a rank 1 parabolic subgroup; in the second $F_{1} \cap F_{2}=\{i d\}$.

Proof. Since the groups $F_{1}$ and $F_{2}$ are peripheral, by theorem I and Susskind's theorem either $\Lambda\left(F_{1}\right) \cap \Lambda\left(F_{2}\right)=\emptyset$ and $F_{1} \cap F_{2}=\{i d\}$, or $F_{1} \cap F_{2}$ is a rank one parabolic subgroup and $\Lambda\left(F_{1}\right)$ and $\Lambda\left(F_{2}\right)$ are tangent. Thus, either $\phi\left(F_{1}\right) \cap \phi\left(F_{2}\right)=\{i d\}$, or $\phi\left(F_{1}\right) \cap \phi\left(F_{2}\right)$ is a rank 1 parabolic subgroup. Using Susskind's theorem again, $\Lambda\left(\phi\left(F_{1}\right)\right) \cap \Lambda\left(\phi\left(F_{2}\right)\right)$ is either empty or a single point.

Proof of Proposition 5.2. Suppose that $F_{0} \in \mathcal{F}_{0}(G)$ and $\phi\left(F_{0}\right) \notin \mathcal{F}_{0}\left(G^{\prime}\right)$; that is, $\phi\left(F_{0}\right)$ is not peripheral. Then there are points of $\Lambda\left(G^{\prime}\right)$ both inside and outside $\Lambda\left(\phi\left(F_{0}\right)\right)$.

Let $\Sigma(G)$ denote the invariant augmented component of $G$. Clearly $\partial \Sigma(G)=\Lambda(G)$. Now $\Sigma(G)$ is a union of discs $\Delta_{i}$ for some collection of $F_{i} \in \mathcal{F}_{0}(G)$. Set $J=\left\{F_{i}: \Delta\left(F_{i}\right) \subset \Sigma(G)\right\}$ and let $B_{\phi}=\cup_{F_{i} \in J} \Lambda\left(\phi\left(F_{i}\right)\right)$.

By corollary $5.4, B_{\phi}$ is a union of mutually tangent circles. Since $\Sigma(G)$ is invariant, it follows that $\bar{B}_{\phi}=\Lambda\left(G^{\prime}\right)$.

Thus we can find $F, F^{\prime} \in J$ and $\xi \in \Lambda(\phi(F)), \xi^{\prime} \in \Lambda\left(\phi\left(F^{\prime}\right)\right)$ with $\xi$ inside $\Lambda\left(\phi\left(F_{0}\right)\right)$ and $\xi^{\prime}$ outside $\Lambda\left(\phi\left(F_{0}\right)\right)$. Now since both $F$ and $F^{\prime}$ are in $J$, we have $\Delta(F) \equiv \Delta\left(F^{\prime}\right)$ and there is a finite chain of groups $F=F_{1}, F_{2}, \ldots, F_{k}=F^{\prime} F_{i} \in J$, such that $\Lambda\left(F_{i}\right)$ and $\Lambda\left(F_{i+1}\right)$ are mutually tangent, $i=1, \ldots, k-1$.

Again applying corollary 5.4, the circles $\Lambda\left(\phi\left(F_{i}\right)\right)$ and $\Lambda\left(\phi\left(F_{i+1}\right)\right)$ are also mutually tangent, $i=1, \ldots, k-1$, and furthermore none of the $\Lambda\left(\phi\left(F_{i}\right)\right)$ can intersect $\Lambda\left(\phi\left(F_{0}\right)\right)$ in more than one 
point. Hence we can find a triple of circles $\Lambda\left(\phi\left(F_{0}\right)\right), \Lambda\left(\phi\left(F_{r}\right)\right), \Lambda\left(\phi\left(F_{r+1}\right)\right)$ with a common point of tangency. Susskind's theorem then tells us that the circles $\Lambda\left(F_{0}\right), \Lambda\left(F_{r}\right)$ and $\Lambda\left(F_{r+1}\right)$ must also have a common point of tangency, but this is impossible since the three groups $F_{0}, F_{r}$ and $F_{r+1}$ are all in $\mathcal{F}_{0}(G)$.

Proof of Theorem III. Let $F \in \mathcal{F}_{0}(G)$ and let $\Delta(F)$ be its invariant disc in $\Omega(G)$. By proposition $5.2, \phi(F) \in \mathcal{F}_{0}\left(G^{\prime}\right)$ and so we can find an associated disc $\Delta(\phi(F)) \subset \Omega\left(G^{\prime}\right)$. There is clearly a unique conformal (or anti-conformal) homeomorphism $\Phi$ of $\Delta(F)$ onto $\Delta(\phi(F)$ ) compatible with the actions of $F$ and $\phi(F)$. Since $\Delta(F)$ and $\Delta(\phi(F))$ are precisely invariant under $F$ and $\phi(F)$ in $G$ and $G^{\prime}$ respectively, $\Phi$ extends equivariantly to all images of $\Delta(F)$ under $G$. Repeating the argument for each $F \in \mathcal{F}_{0}(G)$ and using the fact, proved in theorem I, that $\Omega(G)=\cup_{F \in \mathcal{F}_{0}(G)} \Delta(F)$, we obtain a map $\Phi$ of $\Omega(G)$ into $\Omega\left(G^{\prime}\right)$ inducing $\phi$.

We now want to apply Marden's isomorphism theorem ([5] theorem 8.1) to conclude that $\phi$ is induced by a conjugacy in $\operatorname{PSL}(2, \mathbf{C})$. By theorem II, $G$ is geometrically finite. We have not checked the hypothesis in the isomorphism theorem which requires that the induced homeomorphism have the same orientation on each component; however, as observed by Marden and Maskit, [6], the proof does not use this hypothesis in an essential way. (See also [2], theorem 13.9.) All that is necessary is that $\Phi$ have the same orientation type on adjacent components of $\Omega$. If $\Delta(F)$ and $\Delta\left(F^{\prime}\right)$ are adjacent then $F$ and $F^{\prime}$ contain a common parabolic which forces the orientation type to be the same in both components. We may therefore apply Marden's theorem to conclude that $\Phi$ is a conformal (or anticonformal) homeomorphism of $\hat{\mathbf{C}}$.

\section{References}

[1] L. Greenberg. Fundamental polyhedra for Kleinian groups. Ann. Math., 84:433-441, 1966.

[2] J. Hempel. 3-Manifolds, volume 86 of Annals of Math. Studies. Princeton University Press, 1976.

[3] L. Keen and C. Series. Pleating coordinates for the Maskit embedding of the Teichmüller space of punctured tori. Preprint.

[4] L. Keen and C. Series. The Riley slice of Schottky space. Preprint.

[5] A. Marden. The geometry of finitely generated Kleinian groups. Ann. Math., 99:383-462, 1974.

[6] A. Marden and B. Maskit. On the isomorphism theorem for Kleinian groups. Inventiones Math., 51:9-14, 1979.

[7] B. Maskit. Decomposition of certian Kleinian groups. Acta Math., 130:243-263, 1973.

[8] B. Maskit. Moduli of marked Riemann surfaces. Bull. A.M.S., 80:773-777, 1974.

[9] B. Maskit. Parabolic elements in Kleinian groups. Ann. Math., 117:659-668, 1983.

[10] B. Maskit. Kleinian Groups. Springer, New York, 1987.

[11] B. Maskit and G. Swarup. Two parabolic generator Kleinian groups. Israel J. Math., 64:257-266, 1988. 
[12] D. McCullough. Compact submanifolds of 3-manifolds with boundary. Quart. J. Math., 37:299-307, 1986.

[13] K. Ohshika. Geometrically finite Kleinian groups and parabolic elements. Preprint.

[14] R. Riley. Holomorphically parametrized families of subgroups of SL(2,C). Mathematika, 32:248-264, 1985.

[15] G. P. Scott. Compact submanifolds of 3-manifolds. J. London Math. Soc., 7(2):246-250, 1973.

[16] P. Susskind. Kleinian groups with intersecting limit sets. J. Analyse Math., pages 26-38, 1989.

[17] W. P. Thurston. The geometry and topology of three-manifolds. Unpublished manuscript.

[18] W. P. Thurston. Hyperbolic structures on 3-manifolds I: Deformation of acylindrical manifolds. Ann. Math., 124:203-246, 1986.

[19] D. J. Wright. The shape of the boundary of Maskit's embedding of the Teichmüller space of once punctured tori. Preprint. 